\title{
RECENT ADVANCES IN ACOUSTIC HEATING
}

\author{
P. ULMSCHNEIDER \\ Institut für Theoretische Astrophysik, \\ Universität Heidelberg, \\ Im Neuenheimer Feld 561 \\ D-6900 Heidelberg, Federal Republic of Germany
}

\begin{abstract}
Turbulent surface convection zones of stars generate acoustic waves which contribute to the heating of chromospheres and coronae. The dissipation of limiting strength acoustic shock waves agrees well with the empirically determined chromospheric radiation loss rates. Acoustic waves with frequency and energy required for the chromospheric heating are observed in the solar atmosphere. Acoustic heating can explain the basal chromospheric emission of slowly rotating stars and constitutes a weak background in faster rotating stars; it can not explain the emission-rotation correlation and the surface variation of emission which are due to magnetic heating.
\end{abstract}

\section{Acoustic heating theory and limiting shock strength}

For late-type stars the acoustic heating theory works as follows: acoustic waves generated in the surface convection zone run down the steep density gradient of the outer stellar atmosphere, and, due to energy conservation, grow to large amplitude and form shocks. Shock dissipation heats the outer layers to high temperatures. From the turbulent nature of the convection zone it is clear that acoustic waves must always be generated. Due to the large density decrease in the outer stellar layers even very small acoustic disturbances will be amplified to sizeable amplitudes higher in the atmosphere. It is thus clear that acoustic heating is always present. The acoustic heating mechanism also works for early-type stars where surface convection zones no longer exist. Here the intense radiation field amplifies small acoustic disturbances until strong acoustic shock waves develop. For recent, more detailed reviews of acoustic heating see Narain and Ulmschneider (1989) and Ulmschneider (1990).

Bohn (1984) has computed the theoretical acoustic energy generation rates in the turbulent stellar surface convection zones. These calculations, which have been criticised recently because the buoyancy forces have not been properly taken into account in the treatment of turbulence, depend on three parameters: effective temperature $T_{\text {eff }}$, gravity $g$, and the mixing length parameter $\alpha$. Bohn's results exhibit a strong increase towards higher $T_{\text {eff }}$. This is because the acoustic energy generation depends on a high power of the convective velocity $u$. In fairly efficient convection zones one has $\sigma T_{e f f}^{4} \approx \rho u^{3}$. As the convective velocity of stars is greatest near the surface where the density is lowest, the acoustic energy generation peaks near the F-stars, before the convection zones disappear towards earlier spectral type. After Bohn (1984), the acoustic energy generation also increases when going from dwarfs to giants, because the density in the atmospheres of giant stars is much smaller than in dwarfs, and thus requires larger convective velocities to transport the same total flux $\sigma T_{e f f}^{4}$.

E. R. Priest and V. Krishan (eds.), Basic Plasma Processes on the Sun, 231-235.

(C) 1990 IAU. Printed in the Netherlands. 
In his thesis Bohn (1981, Univ. Würzburg, Germany, unpublished) has computed the acoustic frequency spectra of main-sequence stars (see Fig. 2 of Ulmschneider 1990). These spectra extend roughly over a period range of $P_{A} / 10<P<P_{A}$, where $P_{A}=4 \pi c_{S} /(\gamma g)$ is the acoustic cut-off period. $\gamma$ is the ratio of specific heats, $c_{S}$ the sound speed. As acoustic waves of shorter periods form shocks and dissipate in the low photosphere, this spectral range should not be much affected by the above mentioned criticism. The maximum of the acoustic spectrum is found near $P_{A} / 10$ and shifts towards $P_{A} / 5$ and longerwards for late-type dwarf stars. Thus acoustic waves typically have wave periods of $P=P_{A} / 5$ to $P_{A} / 10$.

In an acoustic wave calculation in the solar atmosphere there are two effects which severely influence the behaviour of the waves. First, the acoustic wave energy flux is strongly affected by radiation damping, when the wave propagates through the radiation damping zone, which in the sun extends to heights of about $200 \mathrm{~km}$, but for other stars can be much more extended (Ulmschneider 1988). Second, it is a persistent result of time-dependent acoustic wave calculations that acoustic shock waves, once formed, tend to quickly reach limiting shock strength. This behaviour, where the wave amplitude becomes essentially constant with height, and independent of the initial amplitude, results from the balance of shock dissipation which decreases the wave amplitude, and amplitude growth which is caused by the steep density gradient. In an isothermal atmosphere the limiting shock strength is given by (Ulmschneider 1970,1989 )

$$
M_{S}^{L i m}=1+\frac{\gamma g}{4 c_{S}} P
$$

From wave periods discussed above one finds $M_{S}^{L i m}=1.3,1.6$ for $P=P_{A} / 10, P_{A} / 5$, respectively. Note that this result is roughly valid for all late-type stars, independent of gravity and $T_{\text {eff }}$. Consequently the velocity-, temperature- and pressure amplitudes of limiting acoustic shock waves are also roughly the same for all late-type stars. With $v \approx$ $2 c_{S}\left(M_{S}^{L_{i m}}-1\right) /(\gamma+1) \approx 0.23 c_{S}$ one finds $F_{M}^{L i m}=\rho v^{2} c_{S} / 3 \approx 2.4 \cdot 10^{4} p$ for $P=P_{A} / 10$, and $F_{M}^{L i m} \approx 9.2 \cdot 10^{4} p$ for $P=P_{A} / 5(P \approx 22,44 s$ for the sun). These limiting acoustic wave fluxes, which are roughly valid for all late-type stars, have been plotted in Fig. 1. In addition Fig. 1 shows the net radiative cooling rate $\Phi_{R}$ and empirical heating flux $F_{M}$ which were recently determined by Anderson and Athay (1989) using semiempirical chromosphere models. It is seen that there is a relatively good agreement between the empirical heating fluxes and the limiting shock strength results, both showing the same pressure dependence.

\section{Solar observations of acoustic waves}

The recent extensive work by Endler and Deubner (1983), Deubner (1988) and Deubner et al. (1988) shows that propagating acoustic waves are present in the solar atmosphere. The success of this group is mainly due to the extensive use of Fourier analysis methods in the data handling, to the data acquisition using long time series by which spectral resolution is greatly improved, and due to the powerful method to remove from the data seeing effects caused by the earth's atmosphere. In a typical observation by Deubner et al. a spectrograph with a slit in equatorial direction is used to observe simultaneously narrow spectral bands with e.g. the lines CaII $8542 \AA$, FeI $8496 \AA$ at the center of the solar disk. Cross correlating the observations in different lines allows to compute the cross power spectrum $C P(\omega)$ and the phase spectrum $\Delta \phi(\omega)$. By removing the influence of seeing, Endler and Deubner (1983) showed on the basis of their corrected phase spectra that there are propagating acoustic waves in the solar atmosphere with periods as low as $40 \mathrm{~s}$. The authors state that this limit 
is a detection limit caused by the great width of the line contribution functions relative to the wavelength of the acoustic waves and does not represent a high frequency limit of solar acoustic waves. To derive numerical values of the acoustic flux expressions like

$$
F_{M} \approx \overline{\rho v^{2} c_{S}} \approx \bar{\rho} \int C P_{S C M}(\omega) c_{G} d \omega
$$

have been used by Deubner et al. (1988). Here $\bar{\rho}$ is a mean density between the two lineforming layers, $c_{G}$ is the group speed which is essentially the sound speed, and $C_{S C M}(\omega)$ is the cross power, corrected for the three important effects of seeing, coherence and modulation transfer function (for a more detailed description of these corrections see also Ulmschneider 1990). Deubner (1988) finds for the low and middle photosphere acoustic fluxes of $F_{M}=2.0 \cdot 10^{7} \mathrm{erg} \mathrm{cm} \mathrm{cm}^{-2} \mathrm{~s}^{-1}$, for the height of NaI $5896 \AA, F_{M}=1.2 \cdot 10^{6} \mathrm{erg} \mathrm{cm}^{-2} \mathrm{~s}^{-1}$ and for the height of CaII $8542 \AA$ roughly $F_{M}=4.5 \cdot 10^{5} \mathrm{erg} \mathrm{cm}^{-2} \mathrm{~s}^{-1}$. He notes that these values tend to be lower bounds because the acoustic flux of short period waves, where seeing overcomes the solar signal, is not included. Taking his estimates of the heights of these lines, $300 \mathrm{~km}, 800 \mathrm{~km}$ and $1500 \mathrm{~km}$, respectively, the acoustic fluxes have been plotted in Fig. 1 . In view of the uncertainties these empirical fluxes agree relatively well with the empirical heating fluxes of Anderson and Athay (1989) and with the limiting shock strength results.

\section{Acoustic heating and stellar observations}

Solar observations indicate that the areas which show the largest chromospheric emission e.g. in the $\mathrm{Ca}$ II $\mathrm{H}+\mathrm{K}$ line cores, are strongly correlated with the magnetic regions at the supergranulation boundaries and with plage regions. Stellar observations (e.g. Rutten 1987) show, that stars can have up to a factor of ten different $\mathrm{Ca}$ II emission fluxes which implies a corresponding difference in the magnetic flux coverage. Stars having similar $T_{\text {eff }}$ and $g$ are thus shown to have a considerable chromospheric emission variability. This variability is attributed to rotation. Stars with high rotation rates are thought to generate much magnetic flux, while stars with low rotation rates generate little magnetic flux and thus little chromospheric emission. Neither the stellar chromospheric variability nor the variation of the chromospheric emission across the solar surface can be explained by acoustic heating which predicts a unique value for the heating flux for a given $T_{\text {eff }}$ and $g$. Here magnetic heating operates (see Narain and Ulmschneider 1989).

An interesting idea particularly by Schrijver (1987) was to separate the chromospheric emission into two components: a nonmagnetic component which is independent of rotation and only depends on $T_{\text {eff }}$ and possibly slightly on $g$, and a magnetic field related component which depends on rotation. The separation of the two components can be achieved using the correlation of the magnetic component with the magnetically dominated X-ray emission. For late-type stars the heating by the nonmagnetic component leads to a basal chromospheric emission flux which may be due to acoustic heating. This basal flux constitutes a low background emission observable in stars of very low rotation rate. For faster rotating stars the acoustic component is usually greatly exceeded by the more energetic magnetic heating component.

The idea, that in the heating of chromospheres two components, a basal nonmagnetic, probably acoustic component and a magnetic, rotation dependent component are at work, can explain several other observations. The low chromospheric variability of the F-stars may be explained as the addition of a strong acoustic heating component to a given variable magnetic heating component. Red giant stars, due to their low rotation rate resulting from angular momentum conservation during the large evolutionary increase in radius and from 
angular momentum loss by massive stellar winds, are another class of stars, where the two component chromospheric heating theory can be tested. Middelkoop (1982, Fig. 4ac) showed that the chromospheric emission variability decreases very much toward late spectral type and there becomes a low basal emission. Here only the nonmagnetic acoustic heating component seems to be present.

\section{REFERENCES}

Anderson, L.S., Athay, R.G..: 1989, Astrophys. J., in press

Bohn, H.U.: 1984, Astron. Astrophys. 136, 338

Deubner, F.-L.: 1988, in Pulsation and Mass Loss in Stars, R. Stalio, L.A. Willson Eds., Kluwer Academic Publ., p. 163

Deubner, F.-L., Reichling, M., Langhanki, R.: 1988, in Advances in Helio- and Asteroseismology, IAU Symp. 123, J. Christensen-Dalsgaard, S. Frandsen Eds., p. 439

Endler, F., Deubner, F.-L.: 1983, Astron. Astrophys. 121, 291

Middelkoop, F.: 1982, Astron. Astrophys. 113, 1

Narain, U., Ulmschneider, P.: 1989, Space Science Reviews, in press

Rutten, R.G.M.: 1987, Astron. Astrophys. 177, 131

Schrijver, C.J.: 1987, in Cool Stars, Stellar Systems and the Sun, J.L. Linsky, R.E. Stencel Eds., Lecture Notes in Physics 291, Springer, Berlin, Germany, p. 135

Ulmschneider, P.: 1970, Solar Phys. 12, 403

Ulmschneider, P.: 1988, Astron. Astrophys. 197, 223

Ulmschneider, P.: 1989, Astron. Astrophys. 222, 171

Ulmschneider, P.: 1990, in Cool Stars, Stellar Systems and the Sun, G. Wallerstein Ed., to be published

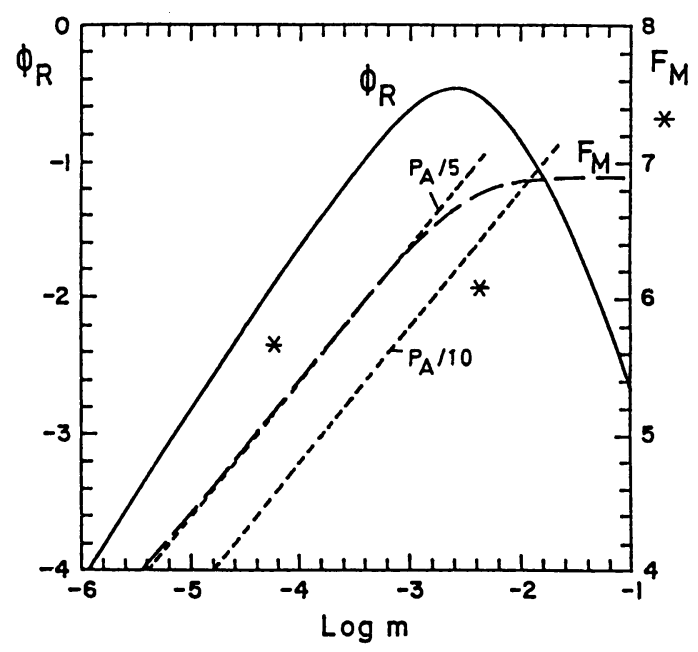

Fig. 1 Net radiative cooling rate $\Phi_{R}\left(\mathrm{erg} \mathrm{cm} \mathrm{cm}^{-3} \mathrm{~s}^{-1}\right)$ and mechanical Flux $F_{M}\left(\mathrm{erg} \mathrm{cm}^{-2}\right.$ $\left.\mathrm{s}^{-1}\right)$ versus mass column density $\mathrm{m}\left(\mathrm{g} / \mathrm{cm}^{2}\right)$ after Anderson and Athay (1989), together with theoretical limiting strength acoustic fluxes $F_{M}^{L I M}$, labeled $P_{A} / 5, P_{A} / 10$, and directly observed acoustic wave fluxes by Deubner (1988), labeled by *. 


\section{DISCUSSION}

ROBERTS: Your comments about a limiting shock strength and basal acoustic heating presuppose that the star's magnetic canopy is sufficiently high that the sound wave has time to propagate unaffected by magnetism. In active stars, the magnetic canopy will be very low and sound waves are therefore more likely to reach that height before they have had time to reach limiting strength. Thereafter, the interaction of sound with the magnetic canopy has to be considered.

ULMSCHNEIDER: That is correct. But in these stars acoustic heating is insignificant. They could modify the magnetic heating rate, however.

VENKATAKRISHNAN: Uchida \& Bappu have a scenario for production of magnetic fields by interaction of convection with the differential rotation caused by spinning up of the contracting core of $\mathrm{G}$ and $\mathrm{K}$ giants. Hence they show that surface rotation does not necessarily mean zero magnetic field.

ULMSCHNEIDER: Stars in the red giant region have very different internal structure due to the fact that they are in different evolutionary states. If the dredging up of magnetic fields from the core was important, then one would expect different magnetic fields on the stars in the same region of the HR Diagram. This would show itself as a variability of the chromospheric emission for these stars. Observations show that all these late-type giants have similar chromospheric emission which argues against magnetic fields and thus for an acoustic heating mechanism.

DALSGAARD: You demonstrated that the limiting strength shocks account for the acoustic flux required to heat the chromosphere. But surely a necessary condition must be that the convection zone produces sufficient acoustic energy to reach this level?

ULMSCHNEIDER: This is correct and both theoretical acoustic energy generation calculations and Deubner's observations show that there appears to be enough energy. Limiting shock strength waves have lost their memory about how they were created. If the wave starts with too much energy, dissipation dominates; with too little energy, amplitude growth prevails. Thus when the limiting shock strength is reached in the middle and high chromosphere then the acoustic flux can be predicted independently of the original source.

SIVARAM: (i) Could you elaborate on how the radiation field amplifies acoustic waves in early-type stars?

(ii) What are the relative magnitudes of acoustic and magnetic heating and at what values of the field does one exceed the other?

ULMSCHNEIDER: (i) Radiative amplification is the opposite of radiative damping. If at the point of maximum compression in the wave cycle you add energy (by absorbing photons due to an increased opacity, for example) the pressure amplitude will increase and the wave will be amplified. If you lose energy (by emitting photons) at that phase you damp the energy.

(ii) That depends on the amount of magnetic flux on the star (or the rotation rate) and on the efficiency of mhd wave energy generation in magnetic flux tubes. 